\title{
First Report of Luminous Stimuli Eliciting Sound Production in Weevils
}

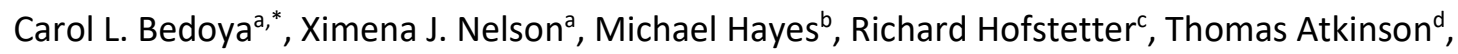 \\ Eckehard G. Brockerhoff ${ }^{a, e}$.
}

${ }^{a}$ School of Biological Sciences, University Of Canterbury, Private Bag 4800, Christchurch, New Zealand; ${ }^{b}$ Department of Electrical and Computer Engineering, University Of Canterbury, Private Bag 4800, Christchurch, New Zealand; 'School of Forestry, Northern Arizona University, Flagstaff AZ, USA; ${ }^{d}$ College of Natural Sciences, University of Texas, USA; ${ }^{\text {SScion }}$ (New Zealand Forest Research Institute), P.O. Box 29237, Christchurch 8540, New Zealand.

*Corresponding author, email: carol.bedoya@pg.canterbury.ac.nz, +64220379875.

\begin{abstract}
Light-based stimuli elicited acoustic responses in male Hylesinus aculeatus Say (Curculionidae: Scolytinae: Hylesinina) instantaneously, with $100 \%$ reliability. Stridulations were elicited with a white light beam in a dark environment and recorded with an ultrasonic microphone. Acoustic responses were consistent and, when compared with sounds produced under stressful conditions (i.e. physical stimulation), no significant differences were found. Hylesinus aculeatus possess an elytro-tergal stridulatory organ and acoustic communication is only present in males. This is also the first report of acoustic communication for this species. Instantaneous light-elicited acoustic communication has potential applications in the development of electronic traps, real-time acoustic detection and identification of beetles, border biosecurity, and noise-reduction in acoustic data collection.
\end{abstract}

Key words: Acoustic Communication; Bark Beetle; Insect; Light Stimulus, Sound Production; Stridulation.

\section{Acknowledgements}

This study was funded by the New Zealand Ministry of Business, Innovation, and Employment (MBIE), grant C04X1407, the Better Border Biosecurity Collaboration (b3nz.org) via MBIE Core Funding to Scion, and Catalyst: Seeding funding from the Royal Society of New Zealand (grant CSGFRI1701). 


\section{Introduction}

Eliciting sound production is a crucial step in the development of methods for the detection and identification of species without the need of a sightline or physical contact. This is particularly useful in insects, where some species are small, cryptic, and considered pests or unwanted organisms (Worner and Gevrey 2006). However, inducing acoustic behaviour is not always possible, and this is often the bottleneck in the implementation of acoustic detection and identification protocols (Mankin 2011). Thus, finding novel ways of eliciting sound production is desirable, as this can aid in technological development for behavioural and ecological studies.

In 1972, Rudinsky and Michael discovered that male Douglas-fir beetles (Dendroctonus pseudotsugae Hopkins) stridulate in response to the female pheromone. This was the first report of chemically-induced acoustic behaviour in the Insecta (Rudinsky and Michael 1972). Aside from chemical stimulation, sound can also induce acoustic behaviour. Playing pre-recorded signals has been demonstrated to elicit acoustic responses in several insect species (Bailey 2003; Mankin 2011). In addition, abiotic factors like temperature, sunlight, and moonlight can trigger sound production in orthopterans and cicadas (Alexander and Moore 1958; Gogala and Riede 1995; Moore 1993). In coleopterans, a much larger group with widespread stridulatory behaviour (Aiken 1985; Lyal and King 1996), effects of light on acoustic communication have been overlooked until recently, when Silk et al. (2018) reported that light affected sound production in the emerald ash borer Agrilus planipennis Fairmaire.

The Eastern ash bark beetle Hylesinus aculeatus Say is a curculionid (Scolytinae: Hylesinina). It feeds on green and white ash (Fraxinus spp.) where adults carve egg galleries inside the phloem layer (Blackman 1922). Acoustic communication was unknown for this species, although four closelyrelated species, $H$. oregonus, $H$. californicus, $H$. fraxini, and $H$. crenatus, have stridulatory capabilities (Kleine 1921; Vernoff and Rudinsky 1980). Members of the genus Hylesinus possess an elytro-tergal stridulatory organ, which is the most common type of sound production mechanism in weevils (Lyal and King 1996), and only males are known to stridulate (Vernoff and Rudinsky 1980). Hylesinus spp. typically colonise old, injured, or diseased trees (Blackman 1922), although some are considered pests of economically important trees such as olives (Olea spp.) and pistachio (Pistacia vera) (Wood and Bright, 1992).

Here, we present a novel finding in which a light stimulus elicits sound production in a bark beetle. The behaviour was serendipitously discovered when using a torch in a darkened room. In this case, the effect of the stimulus is immediate (i.e., latency $<1 \mathrm{~s}$ ), and acoustic communication can thus be elicited ad libitum by the experimenter. To investigate this phenomenon, we excited $H$. aculeatus with a light beam in a dark environment and measured its acoustic responses. We also compared these responses to the ones elicited by physical stimulation. The sounds of $H$. aculeatus are notably simple and uniform, in comparison to other bark beetle species; hence, we hypothesise $H$. aculeatus has solely one type of stridulatory signal, which is used in different behavioural contexts.

\section{Materials and methods}

Signals were acquired inside a purpose-built soundproof box ( $250 \times 300 \times 100 \mathrm{~mm}, \mathrm{w}, \mathrm{l}, \mathrm{d})$ within a chamber with a translucent layer at the top, permitting manual control of light without interfering with the recording set-up. To record sounds, individuals were placed in complete darkness inside 
the chamber in the soundproof box, on top of a smooth surface, and glued upside down on the antero-dorsal part of the elytra. This procedure keeps the individuals still, but does not restrict any of the abdominal movements needed for stridulation. An ultrasonic microphone (M50, Earthworks Inc., Milford, $\mathrm{NH}$ ) with $3 \mathrm{~Hz}$ to $50 \mathrm{kHz}$ frequency range and flat frequency response was located inside the chamber, $20 \mathrm{~mm}$ from the individual's posterior end. Beetle sounds were recorded using a SD 744T audio recorder (Sound Devices LLC, Reedsburg, WI), at a constant temperature of $23^{\circ} \mathrm{C}$.

A manually-controlled white LED light (InGaN, $110 \mathrm{mcd}$ ), located at a perpendicular angle to the beetle and $15 \mathrm{~cm}$ from the uppermost translucent layer of the chamber, was used to elicit acoustic responses. Once turned on, the LED light was left on until the beetle stopped stridulating. Distress signals were recorded in the same apparatus, but sounds were elicited by physically touching the beetle with a soft brush controlled from outside the chamber. All individuals were sexed by examining the convexity of the frons (Underhill 1951) and recorded from the same position. In total, 12 males and 15 females were tested, and every individual was recorded twice (once per stimulus). See Supplementary Material for more information on housing, testing, and definitions.

Four spectro-temporal stridulation parameters (spectral centroid, dominant frequency, duration, inter-note interval) were extracted, and the average value of each parameter for each individual was used for comparison between stridulatory signals elicited with light and physical stimulation (triggering distress sounds). We performed two-tailed paired t-tests $(\alpha=0.05)$ to evaluate changes of each spectro-temporal parameter depending on stimulus. All analyses were implemented in Matlab 2017a.

\section{Results}

Hylesinus aculeatus sounds consisted of broadband, quasiperiodic, uniform train pulses (Fig. 1), also known as chirps, and were only produced by males. No females were recorded producing sounds, regardless of the type of stimulus applied. The spectral components of these sounds propagated throughout the audible and ultrasonic spectrum, and varied between 2 and $30 \mathrm{kHz}$, with $75 \%$ of the energy concentrated below $10.0 \pm 2.9 \mathrm{kHz}$ (mean $\pm \mathrm{SD}$ ). The number of notes was variable and dependent upon the individual ( $9.0 \pm 1.8$ notes per second). 

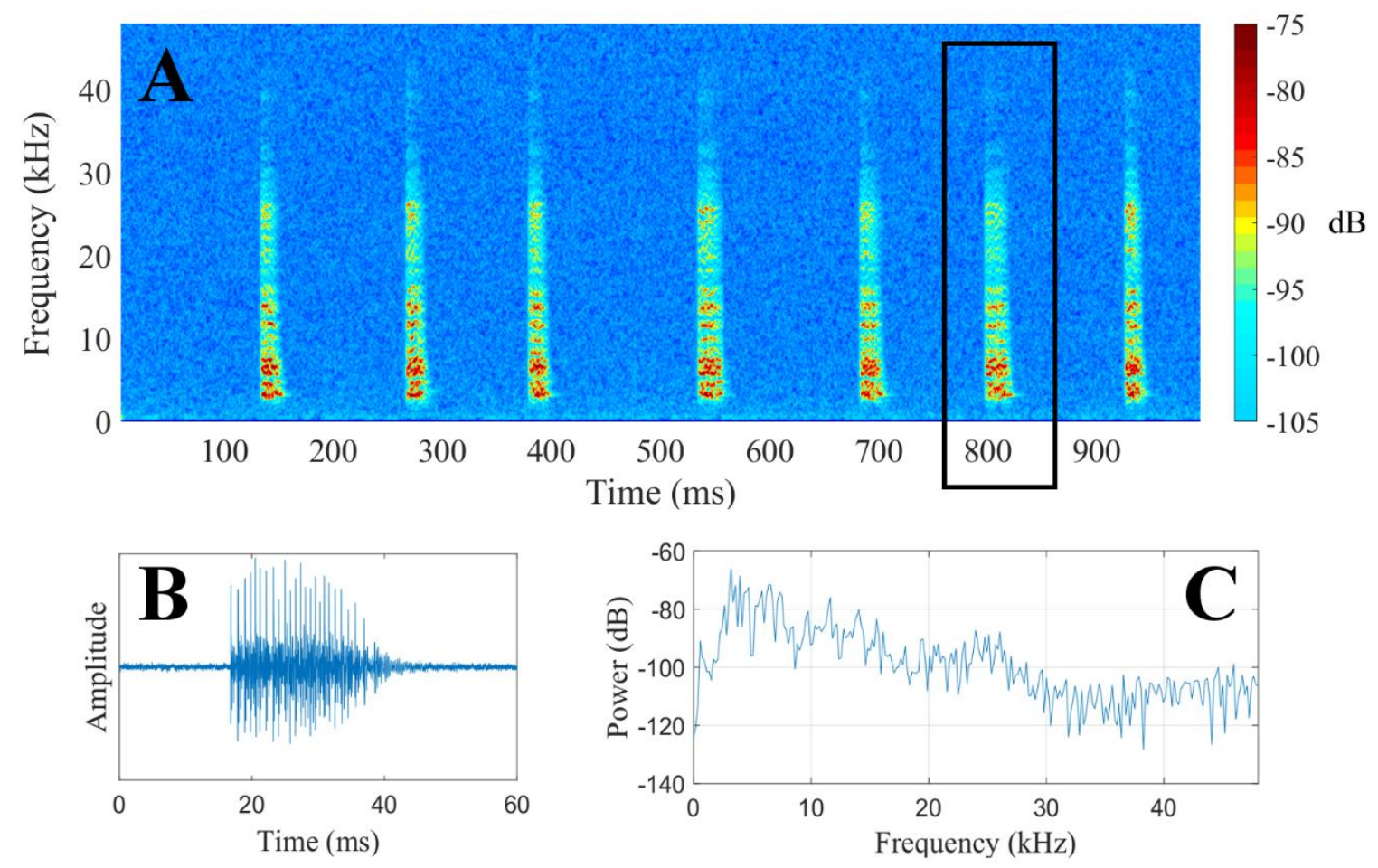

Figure 1 (A) Spectrogram of a train of light-elicited stridulations of a male individual of Hylesinus aculeatus. (B) Time domain representation and (C) power spectrum of the highlighted stridulation in (A).

All male Hylesinus acculeatus stridulated every time the light stimulus was applied, and the stridulatory process began within $0.13 \pm 0.10$ s of the stimulus application. The response length was highly variable, averaging $10.4 \pm 11.0 \mathrm{~s}$, and the spectro-temporal parameters (Table 1 ) were similar to those measured for distress signals (from physical contact). We found no significant differences between stridulations elicited using light and physical stimulation in any of the estimated parameters: spectral centroid $\left(t_{11}=0.41, p=0.685\right)$, dominant frequency $\left(t_{11}=0.42, p=0.681\right)$, duration $\left(t_{11}=-1.02, p=0.327\right)$, and inter-note interval $\left(t_{11}=1.08, p=0.301\right)$.

Table 1 Spectro-temporal parameters (mean $\pm S D$ ) extracted from the stridulations elicited in Hylesinus aculeatus males ( $n=12$ ) with both physical and light stimuli.

\begin{tabular}{|l|cccc|}
\cline { 2 - 5 } \multicolumn{1}{c|}{} & \multicolumn{4}{c|}{ Stridulation Parameter } \\
\cline { 2 - 5 } \multicolumn{1}{c|}{} & Centroid (kHz) & Dominant (kHz) & Duration (ms) & INI (ms) \\
\hline Stimulus & & & & \\
Light & $8.51 \pm 1.78$ & $6.75 \pm 1.37$ & $23.24 \pm 3.18$ & $112.76 \pm 24.19$ \\
Touch & $8.41 \pm 2.21$ & $6.86 \pm 1.50$ & $24.03 \pm 3.82$ & $107.18 \pm 24.34$ \\
\hline
\end{tabular}




\section{Discussion}

This is the first report of light-induced acoustic communication in weevils. All tested male Hylesinus aculeatus responded unequivocally with sound production to both light and physical stimulation. Females, in contrast, were mute and did not stridulate in either scenario; they lack the sclerotisation in the seventh abdominal tergite, which is a common character in other Hylesinus spp. (Rudinsky and Vallo, 1978; Vernoff and Rudinsky, 1980). However, we tested females because some species lacking the plectrum have been found to make sound (Rudinsky and Michael, 1973). Sexually dimorphic acoustic communication in this species is not surprising, as it has been reported for other members of Hylesinus with acoustic communicatory capabilities (Rudinsky and Vallo 1978; Vernoff and Rudinsky 1980), and it is a common trait in weevils with elytro-tergal stridulation (Lyal and King 1996).

The acoustic characteristics of sounds elicited in $\mathrm{H}$. aculeatus by light or physical distress were similar, consisting of a train of simple (i.e., single-note) stridulatory signals, to those of other bark beetles with elytro-tergal organs, such as Dendroctonus beetles (Ryker 1988). However, the spectrotemporal parameters differed from those previously found in other Hylesinus species (Rudinsky and Vallo 1978; Vernoff and Rudinsky 1980). The signals were broadband, with spectral components distributed throughout the audible and ultrasonic spectrum, which is common in species with stridulatory mechanisms (Grant et al. 2014; Yturralde and Hofstetter 2015).

Stridulations elicited with the light stimulus were immediate, enabling complete control of acoustic communication in $\mathrm{H}$. aculeatus. Instantaneous acoustic response to light has not previously been reported for insects. Silk et al. (2018) reported a significant increase in acoustic behaviour of the emerald ash borer Agrilus planipennis associated with the presence of light; although, this was quantified over a 16-hour period (Silk et al. 2018). The instant acoustic response found here also differs from behaviours reported in other insects that use sunlight as an onset cue for acoustic communication, in which the gradient in luminosity is the factor triggering sound production (Alexander and Moore 1958; Gogala and Riede 1995). Because of this, and because H. aculeatus mostly feeds and lives inside the inner bark of ash trees (Blackman 1922), we hypothesise that the function of sound in this species is not associated with any circadian or photoperiodic calling behaviour. Instead, the function of the sound appears to be an anti-depredatory strategy. Bark and ambrosia beetles live most of their lifetime inside plant tissue; therefore, sudden exposure to light means that the gallery has been compromised. The emission of sound could be used as a defensive strategy to deter predators as similar behaviours have been previously reported for other insects (Conner, 2014; Masters, 1979). It could also work as an alarm sound to inform conspecifics, like the vibrational signals produced by termites exposed to bright light after a predator has disturbed their nest (Kirchner et al., 1994).

Light as a trigger of sound production has potential applications in the development of electronic traps, as combining light with acoustic identification methods may yield real-time categorisation of collected specimens. Light could also be used to elicit sound production in environments where physical contact with individuals is difficult to achieve, and where the presence of individuals needs to be detected (e.g., hitchhiker beetles in cargo containers). Another immediate application of this behaviour is the significant improvement in the signal-to-noise ratio during acoustic data 
acquisition. Since light is a contactless way of eliciting sound production, beetles can be recorded in fully-sealed confined environments and the stridulatory process can be controlled ad libitum by externally switching a light.

Eliciting controlled acoustic responses using light is a phenomenon that has been almost completely overlooked in beetles, yet the report of Silk et al. (2018), in conjunction with the present study, suggests that this phenomenon could be common among coleopterans. We encourage others to look further into this as non-contact methods of eliciting ad libitum acoustic communication have numerous practical applications. Our findings may lead to new ideas for combining light and acoustics in detection and identification uses for ecological monitoring, trap design, and border biosecurity applications.

\section{References}

Aiken RB (1985) Sound production by aquatic insects. Biol Rev 60:163-211.

Alexander RD, Moore TE (1958) Studies on the acoustical behavior of seventeen-year cicadas (Homoptera: Cicadidae: Magicicada). Ohio J Sci 58:107-127.

Bailey WJ (2003) Insect duets: underlying mechanisms and their evolution. Physiol Entomol 28:157174.

Blackman MW (1922) Mississippi bark beetles. Mississippi Agricultural Experiment Station, Technical Bull 11:62-63.

Conner WE (2014) Adaptive Sounds and Silences: Acoustic Anti-Predator Strategies in Insects. In: Hedwig B (eds) Insect Hearing and Acoustic Communication. Animal Signals and Communication, vol 1. Springer, Berlin, Heidelberg, 65-79.

Grant PBC, Roets F, Samways MJ (2014) Sound characterization and structure of the stridulatory organ in Gonogenia tabida (Coleoptera: Carabidae). Bioacoustics 23:209-217.

Gogala M, Riede K (1995) Time sharing of song activity by cicadas in Temengor Forest Reserve, Hulu Perak, and in Sabah, Malaysia. MNJ 48:297-305.

Kirchner WH, Broecker I, Tautz J (1994) Vibrational alarm communication in the damp-wood termite Zootermopsis nevadensis. Physiol Entomol 19:187-190.

Kleine R (1921) Der Stridulationsapparat der Ipidae II. Entomologische Blätter 17:22-26.

Lyal CHC, King T (1996) Elytro-tergal stridulation in weevils (Insecta: Coleoptera: Curculionoidea). J Nat Hist 30:703-773.

Mankin RW, Hagstrum DW, Smith MT, Roda AL, Kairo MTK (2011) Perspective and promise: a century of insect acoustic detection and monitoring. Am Entomol 57:30-44.

Masters WM (1979) Insect disturbance stridulation: Its Defensive role. Behav Ecol Sociobiol 5:187200.

Moore TE (1993) Acoustic signals and speciation in cicadas (Insecta: Homoptera: Cicadidae). In: Lees DR, Edwards D (eds) Evolutionary Patterns and Processes. Linnean Society Symposium no. 14, London: Academic Press, 269-284. 
Ryker L (1988) Acoustic studies of Dendroctonus bark beetles. Fla Entomol 71:447-461.

Rudinsky JA, Michael RR (1972) Sound production in Scolytidae: Chemostimulus of sonic signal by the Douglas-fir beetle. Science 175:1386-1390.

Rudinsky JA, Michael RR (1973) Sound production in Scolytidae: Stridulation by female Dendroctonus beetles. Journal of Insect Physiology 19:689-705.

Rudinsky JA, Vallo V (1978) The ash bark beetles Leperisinus fraxini and Hylesinus oleiperda: stridulatory organs, acoustic signals, and pheromone production. Z Angew Entomol 87:417-429.

Silk PJ, Roscoe LE, Brophy M, Price J, Ryall KL (2018) Influence of light on sound production behaviors in the Emerald ash borer, Agrilus planipennis. Entomol Exp Appl eea.12727.

Underhill RA (1951) Life history and habits of Leperisinus californicus Swaine and Leperisinus oregonus Blackman with a revision of the genus Leperisinus of North America north of Mexico. PhD thesis, Oregon State University 48-51.

Vernoff S, Rudinsky JA (1980) Sound production and pairing behavior of Leperisinus californicus Swaine and L. oregonus Blackman (Coleoptera: Scolytidae) attacking Oregon ash. Z Angew Entomol 90:58-74.

Wood SL, Bright DE (1992) A catalog of Scolytidae and Platypodidae (Coleoptera), Part 2. Taxonomic Index Volume A. Great Basin Naturalist Memoirs 13:1-1533.

Worner SP, Gevrey M (2006) Modelling global insect pest species assemblages to determine risk of invasion. J Appl Ecol 43:858-867.

Yturralde KM, Hofstetter RW (2015) Characterization of stridulatory structures and sounds of the larger Mexican pine beetle, Dendroctonus approximatus (Coleoptera: Curculionidae: Scolytinae). Fla Entomol 98:516-527. 


\section{SUPPLEMENTARY MATERIAL}

1) Definitions

Dominant Frequency: Frequency component that carries the largest amount of energy.

Spectral centroid: Analogous feature to the centre of mass in mechanical systems. It represents the frequency in which the centroid of the power spectral distribution is located.

Note: Is the main subunit of the stridulatory sound, also known as chirp, produced by the beetle. Chirps might be composed by a single note or multiple notes, separated by periods of silence known as inter-note-intervals. Hylesinus aculeatus produces simple chirps, which consist of a single note.

Note Duration: Length of a note within a stridulatory sound.

Inter-note-interval: Interval between two consecutive notes within the same chirp, measured from the end of one note to the beginning of the consecutive note. In species whose chirps consist of a single note, the inter-note-interval is equivalent to the inter-chirp-interval.

\section{2) Additional Material and Methods}

\section{Beetle collection and housing}

Beetles were collected in Austin, TX, USA (30.2494 N, $97.6998 \mathrm{~W})$ from emergence chambers with specimens of green ash, Fraxinus pennsylvanica. Individuals were collected as they emerged and stored in a container with moist paper towel to keep the beetles hydrated, inside a refrigerator at $3^{\circ} \mathrm{C}$.

\section{Time delay estimation}

The time lag between the application of the light stimulus and the acoustic response was estimated using an accelerometer (352A24, PCB piezoelectronics, Depew, NY, USA) attached to the LED light that generated the stimulus. The accelerometer and the ultrasonic microphone (M50) were both connected to the same recording device (SD 744T). The action of the finger touching the switch to turn the light on produced a small spike in one of the channels, which was enough to measure the delay in the response since both channels were synchronised.

\section{Distress stimulus}

Mechanical stimulation was manually controlled from outside the chamber. A thin paintbrush was introduced via a small hole parallel to the beetle and controlled ad libitum by the researcher. Slightly pushing the brush towards the abdomen of the individual was enough to trigger sound production. Each beetle was touched once.

Algorithm parameters 
The short-time Fourier transform (STFT) used for the spectro-temporal analysis of the data was estimated using a rectangular window of 1024 samples and $75 \%$ overlap. 